\title{
Laser-induced fine structures on silicon exposed to $\mathrm{THz}-\mathrm{FEL}$
}

\section{$\operatorname{AUTHOR}(\mathrm{S}):$}

Irizawa, Akinori; Suga, Shigemasa; Nagashima, Takeshi; Higashiya, Atsushi; Hashida, Masaki; Sakabe, Shuji

\section{CITATION:}

Irizawa, Akinori ...[et al]. Laser-induced fine structures on silicon exposed to THz-FEL. Applied Physics Letters 2017, 111(25): 251602.

\section{ISSUE DATE:}

2017-12-18

URL:

http://hdl.handle.net/2433/267219

\section{RIGHT:}

(c) 2017 Author(s). Published by AlP Publishing. This article may be downloaded for personal use only. Any other use requires prior permission of the author and AIP Publishing. This article appeared in kinori Irizawa, Shigemasa Suga, Takeshi Nagashima, Atsushi Higashiya, Masaki Hashida, and Shuji Sakabe, "Laser-induced fine structures on silicon exposed to THz-FEL", Appl. Phys. Lett. 111, 251602 (2017) and may be found at https://doi.org/10.1063/1.5006014. 


\section{Laser-induced fine structures on silicon exposed to THz-FEL}

Cite as: Appl. Phys. Lett. 111, 251602 (2017); https://doi.org/10.1063/1.5006014

Submitted: 21 September 2017 • Accepted: 01 December 2017 • Published Online: 18 December 2017

Akinori Irizawa, Shigemasa Suga, Takeshi Nagashima, et al.

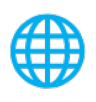

View Online
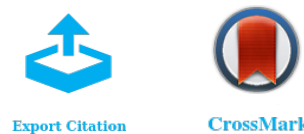

CrossMark

\section{ARTICLES YOU MAY BE INTERESTED IN}

Femtosecond laser-induced periodic surface structures

Journal of Laser Applications 24, 042006 (2012); https:/ / doi.org/10.2351/1.4712658

On the role of surface plasmon polaritons in the formation of laser-induced periodic surface structures upon irradiation of silicon by femtosecond-laser pulses

Journal of Applied Physics 106, 104910 (2009); https://doi.org/10.1063/1.3261734

Pulse number dependence of laser-induced periodic surface structures for femtosecond laser irradiation of silicon

Journal of Applied Physics 108, 034903 (2010); https://doi.org/10.1063/1.3456501

母QBLOX

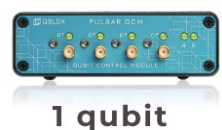

Shorten Setup Time Auto-Calibration More Qubits

Fully-integrated Quantum Control Stacks Ultrastable DC to $18.5 \mathrm{GHz}$ Synchronized $<<1$ ns Ultralow noise

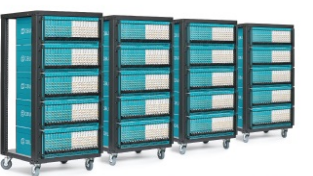

100s qubits

visit our website > 


\title{
Laser-induced fine structures on silicon exposed to THz-FEL
}

\author{
Akinori Irizawa, ${ }^{1, a)}$ Shigemasa Suga, ${ }^{1}$ Takeshi Nagashima, ${ }^{2}$ Atsushi Higashiya, ${ }^{2}$ \\ Masaki Hashida, ${ }^{3}$ and Shuji Sakabe ${ }^{3}$ \\ ${ }^{1}$ The Institute of Scientific and Industrial Research, Osaka University, 8-1 Mihogaoka , Ibaraki, Osaka \\ 567-0047, Japan \\ ${ }^{2}$ Faculty of Science and Engineering, Setsunan University, 17-8 Ikeda-Nakamachi, Neyagawa, \\ Osaka 572-8508, Japan \\ ${ }^{3}$ Advanced Research Center for Beam Science, Institute for Chemical Research, Kyoto University, Gokasho, \\ Uji, Kyoto 611-0011, Japan and Department of Physics, Graduate School of Science, Kyoto University, \\ Kitashirakawa, Sakyo, Kyoto 606-8502, Japan
}

(Received 21 September 2017; accepted 1 December 2017; published online 18 December 2017)

\begin{abstract}
We found the irradiation of focused linearly polarized terahertz $(\mathrm{THz})$-waves emitted from $\mathrm{THz}$ free-electron laser (THz-FEL) engraved fine periodic stripe structures on the surfaces of singlecrystal $\mathrm{Si}$ wafers. The experiments were performed at several wavelengths ranging from 50 to $82 \mu \mathrm{m}$ with a macro-pulse fluence up to $32 \mathrm{~J} / \mathrm{cm}^{2}$. The engraved structures are considered equivalent to the laser-induced periodic surface structures (LIPSS) produced by the irradiation of a femtosecond (fs)-pulsed laser in the near-infrared (NIR) region. However, the minimum period of $\sim 1 / 25$ of the wavelength in the present case of THz-FEL is surely much smaller than those reported so far by use of fs-lasers and no more explicable by the so far proposed mechanisms. The finer LIPSS confirmed by longer-wavelength laser excitation by means of THz-FEL motivates investigation into the universal mechanism of LIPSS formation, which has been under a hot debate for decades. Published by AIP Publishing. https://doi.org/10.1063/1.5006014
\end{abstract}

Laser irradiation processing via coherency is one of the most promising methods for functionalizing surfaces with modifications. Laser-induced periodic surface structure (LIPSS) formation can be used for surface nanostructuring with a spatial scale of less than the laser wavelength. In the case of a fs-laser, it is possible to control the hydrophobicity ${ }^{1}$ and color ${ }^{2}$ of materials by carving LIPSS onto the metal surface. Furthermore, if the wavelength of the laser is changed, various surface structures varying from the micrometer to nanometer size may be controlled and fabricated, and the laser will create more versatile surface functionalities. By exploring advanced physical and chemical interactions between electromagnetic waves and materials, one can expect as the material designing for use in energy conversion and catalysts will progress remarkably. However, although numerous studies were conducted following the first observation of LIPSS by ruby laser irradiation in $1965,{ }^{3}$ several LIPSS formation mechanisms have been proposed and are still being studied because LIPSS must be classified into one of several types depending on the period and direction and in terms of the laser power, pulse duration, wavelength, polarization, and target material.

Low-spatial-frequency LIPSSs (LSFLs) have periods close to the wavelength of fs-laser pulses and are commonly described by models based on an interaction of scattered waves and/or surface plasmon polaritons (SPPs) with the incident laser light. ${ }^{4}$ The models predict the formation of LIPSS perpendicular to the electric field vector of the excitation light at normal incidence. Occasionally, LIPSSs parallel to the laser polarization have been observed. ${ }^{5}$ High-spatial-

\footnotetext{
${ }^{\text {a) }}$ Author to whom correspondence should be addressed: irizawa@sanken. osaka-u.ac.jp
}

frequency LIPSSs (HSFLs) with periods much smaller than the wavelength have sometimes been reported. ${ }^{6}$ For models based on the interaction between the surface wave and the incident laser light, these are unexpected structures. Thus, alternative models, taking into account generation of a second harmonic, ${ }^{6}$ hydrodynamics of molten materials, ${ }^{7}$ and self-organization, ${ }^{8}$ have been proposed. Despite the efforts by researchers on both the experiments and theories described above, no comprehensive understanding of LIPSS formation has yet been obtained.

The interaction or interference between the surface wave and incident laser is thought to be a plausible origin of LSFL. In this case, the dependence of LIPSS formation on the wavelength of the excitation laser must be fully understood to determine the mechanism of LIPSS formation. So far, the wavelength dependence of LIPSS remains unclear because most studies on LIPSS have been performed only in narrow wavelength regions using near-infrared femtosecond pulse lasers with a wavelength of approximately $800 \mathrm{~nm}$ and their second harmonics. On the other hand, FELs emit intense electromagnetic pulses with a tunable wavelength over a rather wide range. THz-FELs, with fluences higher than the damage threshold of materials, have been recently developed. ${ }^{9}$ This means that an intense THz-FEL in a long wavelength limit beyond the existing laser systems can be used for LIPSS experiments.

In this letter, we report the results of experiments in which $\mathrm{Si}$ wafers were irradiated by an intense $\mathrm{THz}$-wave emitted from a THz-FEL with wavelengths ranging from 50 to $82 \mu \mathrm{m}$. We observed LIPSS fabricated under different conditions by THz-wave irradiation, where the LIPSSs parallel to the polarization of the THz-wave were observed. The dependences of the LIPSS period on the wavelength, fluence, 
and number of macropulses of the THz-FEL were fully investigated. The ratio of the minimum LIPSS period observed in this study relative to the excitation wavelength was $\sim 1 / 25$. This ratio is smaller by one order of magnitude than that in conventional experiments with near-infrared (NIR) femtosecond (fs)-lasers.

The experiments were performed using the THz-FEL at the quantum beam research facility at the Institute of Scientific and Industrial Research (ISIR) of Osaka University. ${ }^{9}$ The THz-FEL emits macro-pulses with a duration of $4 \mu \mathrm{s}$ at a repetition rate of $5 \mathrm{~Hz}$ as schematically shown in Fig. 1(a). One macro-pulse is composed of $\sim 108$ micro-pulses with a duration of $20 \mathrm{ps}$ at an interval of $\sim 37 \mathrm{~ns}$. The micro-pulse contains, for example, $\sim 60$ cycles of the THz-wave at a typical wavelength of $100 \mu \mathrm{m}(3 \mathrm{THz})$. The THz wavelength was tuned in this experiment from 50 to $100 \mu \mathrm{m}$. Because the THz-FEL is emitted through an undulator, the emitted THzwave was linearly polarized and monochromatic. The typical spectral line width $\Delta \lambda$ of the THz-wave at wavelength $\lambda$ is $\Delta \lambda \approx 0.05 \lambda$ as shown in the right-hand inset of Fig. 1(b). The energy per macro-pulse of the terahertz wave was measured by using an energy meter manufactured by COHERENT Inc. that was subjected to sensitivity correction in the terahertz band. The energy fluctuation of the THz-FEL used for irradiation was $3 \%-7 \%$ on average.

The THz-FEL wave was focused on the sample at normal incidence by an off-axis paraboloidal mirror with a diameter of $25.4 \mathrm{~mm}$ and a focal length of $25.4 \mathrm{~mm}$. It was confirmed that the THz-wave had an excellent Gaussian profile by knife-edge scanning measurements at the focal point. The wavelength dependence of the beam radius $(2 \sigma)$ at the focal point is displayed in the left-hand inset of Fig. 1(b), which shows that for each wavelength, the THz-wave was focused to a spot size of about double the wavelength. Figure 1(b) shows the achieved fluence of one-macro-pulse as a function of the wavelength, deduced by taking into account the wavelength dependences of the macro-pulse energy and beam diameter on the sample. The maximum fluence of the macro-pulse in this experiment was $\sim 40 \mathrm{~J} / \mathrm{cm}^{2}$ at a wavelength of $\sim 80 \mu \mathrm{m}$, which is equivalent to the average maximum fluence of a micro-pulse of $\sim 0.37 \mathrm{~J} / \mathrm{cm}^{2}$. The solid circles in Fig. 1(b) represent the wavelength and fluence of the excitation wave used in this study. The open-circles at $\lambda=68 \mu \mathrm{m}$ show the values of the fluence reduced by attenuators to perform fluence-dependence experiments. The fluence was controlled by a Teflon plate as attenuators with thicknesses of 1,2 , and $5 \mathrm{~mm}$ inserted just before the paraboloidal mirror. The error bars of the beam radius and fluence were derived from the energy fluctuation of the THz-FEL.

The samples used in the experiment were commercially available high-purity single-crystal Si wafers: one with a surface orientation of (100) and a resistivity of $1 \mathrm{k} \Omega \cdot \mathrm{cm}$ and two with orientations of (111) with resistivities of $1 \mathrm{k} \Omega \cdot \mathrm{cm}$ and $10 \mathrm{k} \Omega \cdot \mathrm{cm}$. The surfaces were mirror-finished products, and no additional cleaning treatment was employed. The experiment was conducted in ambient conditions. Throughout this letter, the results presented are for the Si (111) sample with a resistivity of $1 \mathrm{k} \Omega \cdot \mathrm{cm}$ because there was no significant difference in the formation of LIPSS for the different plane orientations and resistivities of the samples.

Figures 2(a) and 2(b) show optical microscopy images of typical LIPSS formed on the sample for 10 and 50 macropulses. The central wavelength of the irradiated THz-wave was $82 \mu \mathrm{m}$. It should be noted that the direction of the LIPSS was almost parallel to that of the electric field of the THz-wave indicated by the arrows in Fig. 2(a). We confirmed that the directions and periods of the LIPSS remained unchanged regardless of the in-plane rotation of the samples with respect to the direction of the electric field of the THz-wave.

When the number of macro-pulses was small, there was a tentative region where the stripes expanding radially could be recognized in the outer peripheral region, as shown in Fig. 2(a). As the number of macro-pulses increased from 10 to 50 , the radial stripe structures became inconspicuous as shown in Fig. 2(b). The radial structures can be considered as cracks along certain crystal orientations caused by the impact generated by the initial pulse irradiation. Such cracks are only induced for the initial laser pulse beyond a certain power, and they are overwritten and masked as the number of irradiation pulses increases [Figs. S1(a) and S1(b) in the supplementary material]. In the following section, we focus on the dominant LIPSSs that were parallel to the electric field and discuss how the period varied depending on the FEL irradiation conditions.

Figure 2(c) shows a typical scanning electron microscopy (SEM) image of the LIPSS formed with a 10-macropulse irradiation. The stripes protruded from the sample surface. Although the height of the individual stripes depended highly on the position, the typical height of the stripes was estimated to be submicron level using a laser microscope (unpublished). There were noticeably protruding parts of the stripes that correspond to the black dot regions in (a)

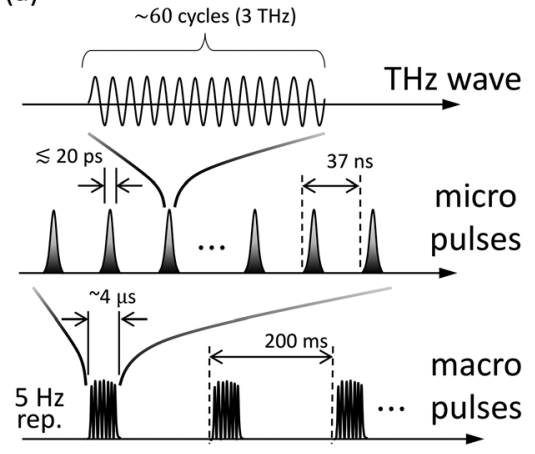

(b)

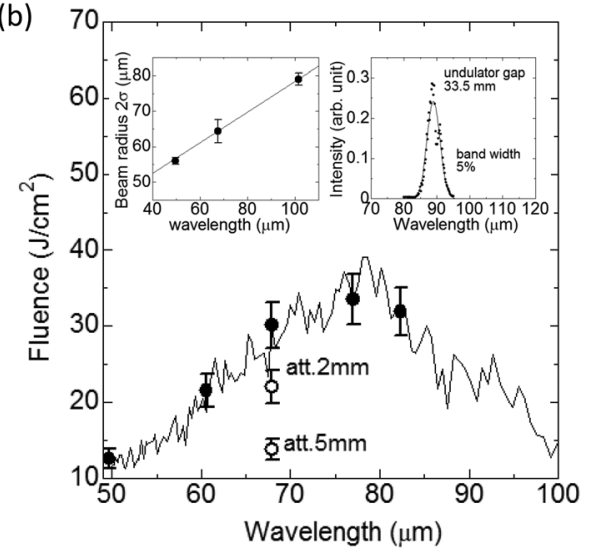

FIG. 1. (a) Macro- and micro-pulse structures of the THz-FEL used in this experiment. (b) Wavelength dependence of the fluence of one-macropulse. Circles with error bars indicate the fluence of the irradiated THz-FEL, filled circles indicate the original, and the open circles indicate the attenuated beams. The left inset shows the beam radius $(2 \sigma)$ at the focal point as a function of wavelength $\lambda$. The right inset shows a typical spectral shape of THz-FEL. 

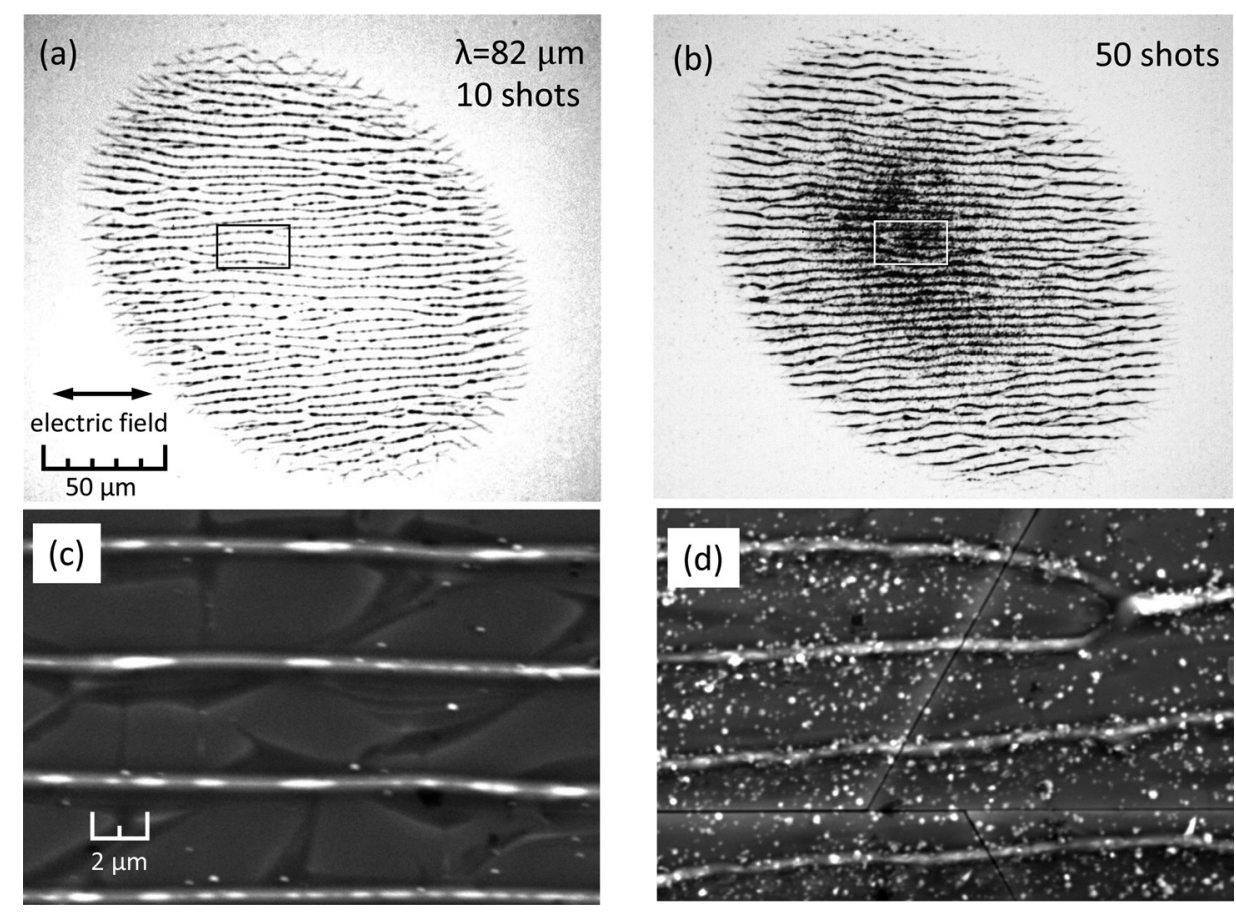

FIG. 2. Optical photomicrographs of LIPSS generated by (a) 10 and (b) 50 macro-pulse irradiation at a wavelength of $82 \mu \mathrm{m}$ with a fluence of $\sim 32 \mathrm{~J} / \mathrm{cm}^{2}$. (c) SEM image of the square area in Fig. 2(a). (d) SEM image of the square area in Fig. 2(b). The deposits shown as white dots can be attributed to the ablated materials. the optical micrograph shown in Fig. 2(a). Those features were considered to be formed by the spouting of ionized $\mathrm{Si}$ particles by Coulomb explosion and/or solidification of melting. The deposits densely dispersed in the vicinity of the center of the LIPSS shown in Figs. 2(b) and 2(d) can be attributed to the ablated materials from the stripes and protruding parts.

The energy required for ionization and/or melting of the sample is naturally transferred from the THz-wave through electronic excitation. Although the photon energy (on the order of $10 \mathrm{meV}$ ) of the THz-wave is much smaller than the bandgap energy $(\sim 1.1 \mathrm{eV})$ of $\mathrm{Si}$, free carriers (electrons and holes) can be generated through impact ionization as this is the most probable process under intense $\mathrm{THz}-$ wave irradiation. ${ }^{10}$ The electric field of the THz-wave accelerating the electrons is so large that only a few tens of femtoseconds are enough to obtain an electron kinetic energy higher than the impact ionization threshold. Then, the density of the free electrons increases as in an avalanche by multiple impact ionizations at the rising edge of the micro-pulse. Consequently, the strong electric field of the subsequent THz-wave extracts the free electrons exponentially from a local area, inducing a Coulomb explosion in a micro-pulse duration. After these nonthermal excitations, melting and/or boiling may occur in the vicinity of the LIPSS and on the ablated particles as a result of energy relaxation during a macro-pulse irradiation. Note that the minimal process necessary to form the LIPSS by THz-FEL should be completed within $\sim 4 \mu$ s of the macro-pulse duration (including 108 micro-pulses) because the LIPSS can be generated by only a single macro-pulse irradiation, as shown in Fig. S1(a) (supplementary material).

As shown in Fig. 2(b), the LIPSS period tends to become narrower around the center than in the periphery region even in the same LIPSS, suggesting that the LIPSS period is dependent on the local irradiation power in the beam spot. This is consistent with the fluence dependence of the LIPSS period as discussed below. In the meantime, the bandwidth of irradiated THz-FEL is about $5 \%$ as mentioned above. Then, it is unlikely that the difference between the center and the periphery of LIPSS periodicity ranging from 20 to $40 \%$ as analyzed later is due to the spatial wavelength distribution of THz-FEL.

Figure 3(a) shows the average LIPSS period as a function of the macro-pulse fluence for a wavelength of $68 \mu \mathrm{m}$ and for 10 macro-pulses. The LIPSS period was evaluated from the average of $10-15$ periods in the vicinity of the center of the beam spot. The LIPSS period was found to remain nearly constant below $28 \mathrm{~J} / \mathrm{cm}^{2}$. Above $30 \mathrm{~J} / \mathrm{cm}^{2}$, there was a significant period decrease relative to that at the lower fluences, demonstrating the non-linearity of the LIPSS period as a function of the irradiation power. The decrease in the LIPSS period with the fluence is also consistent with the observation that the LIPSS period becomes narrower near the center than in the periphery where the local intensity is reduced. As for the intensity of the irradiated lasers, the threshold fluences can be compared between the NIR fs-laser and THz-FEL. In the case of the NIR fs-laser with the pulse duration of $130 \mathrm{fs}$ and the wavelength of $800 \mathrm{~nm},{ }^{11}$ the damage threshold fluence for 100 single-pulses is about $0.15 \mathrm{~J} / \mathrm{cm}^{2}$ (Figs. 4 and 5 in the Ref. 11). In our case at the wavelength of $77 \mu \mathrm{m}$, LIPSS generation by 1 macro-pulse irradiation containing almost 100 micro-pulses with $32 \mathrm{~J} / \mathrm{cm}^{2}$ suggests that the possible damage threshold will be less than $0.3 \mathrm{~J} / \mathrm{cm}^{2}$ [see Fig. S1(a) in the supplementary material]. Since there are great differences in both the pulse duration (130 fs and $20 \mathrm{ps)}$ and the wavelength $(800 \mathrm{~nm}$ and $77 \mu \mathrm{m})$, more detailed and systematic experiments of THz-FEL LIPSS formation with changing the wavelength, laser intensity, and pulse duration will be required in the next step.

Figure 3(b) shows the wavelength dependence of the LIPSS period for LIPSS generated by 10-macro-pulse irradiation at various fluences. The circles, triangles, and squares indicate the LIPSS period at fluences of 13,22 , and $32 \mathrm{~J} / \mathrm{cm}^{2}$, 

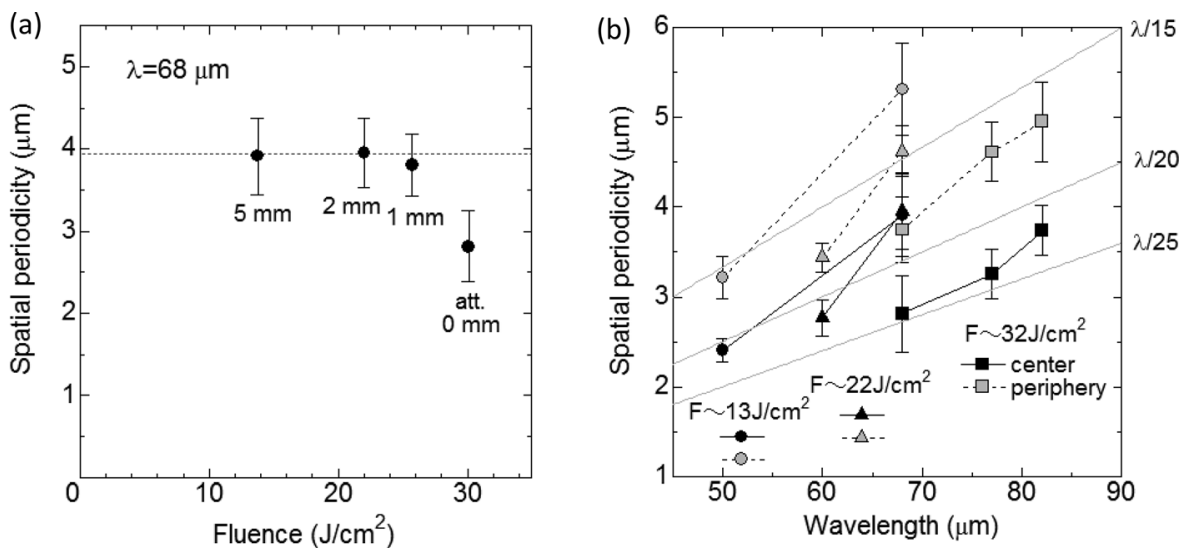

FIG. 3. (a) Dependence of the LIPSS period on macro-pulse fluence at a wavelength of $68 \mu \mathrm{m}$ (in the vicinity of the center with 10-macro-pulse irradiation). (b) Wavelength dependence of the LIPSS period with 10-macro-pulse irradiation at various fluences. Circles, triangles, and squares indicate the LIPSS period for fluences of 13,22 , and $32 \mathrm{~J} / \mathrm{cm}^{2}$, respectively. Black and gray symbols show the period in the vicinity of the center and in the periphery, respectively.

respectively. The black and gray symbols show the period near the center and in the periphery, respectively. As shown in Fig. 3(b), the LIPSS period increases with the wavelength in both the central and the peripheral parts at a fixed fluence. The ratio of the LIPSS period to the wavelength reaches $\sim 1 / 25$ in the minimum case. This value is four times smaller than that reported for HSFL on Si (Ref. 12) by NIR fs-laser irradiation in air or a vacuum. The ratio of the LIPSS period for HSFL can be expressed by the relation $\lambda_{\mathrm{SP}} / \lambda_{\mathrm{L}}=1 / 2 \mathrm{n}$, where $\mathrm{n}$ is the refractive index of the transparent material. ${ }^{13-15}$ In our case, this relation leads to an estimated ratio for the LIPSS period of about $1 / 6.8\left(n_{\mathrm{Si}}=3.41\right),{ }^{16}$ which is much bigger than the experimentally observed value of $1 / 25$.

The LIPSS generated by the THz-FEL mentioned above were (i) parallel to the electric field of the linearly polarized THz-FEL at normal incidence and (ii) extremely fine $(\sim 1 / 25)$ compared with the wavelength. In addition, (iii) the LIPSS period increased almost linearly with the wavelength, while (iv) it decreased nonlinearly with the fluence. These aspects except for (iii) were unexpected if using conventional models based on the interaction and/or interference with the surface wave and incident electromagnetic wave. The model based on thermodynamic instability ${ }^{8}$ predicts that the LIPSS period is insensitive to the wavelength, which is also inconsistent with one of our findings, i.e., the period depends on the $\lambda$.

To accumulate more insights, the change in the LIPSS period $\Delta$ as a function of the number $N$ of irradiated macropulses was investigated. Figures 4(a) and 4(b) are $\ln \Delta-\ln$ $N$ plots for wavelengths of 82 and $68 \mu \mathrm{m}$, respectively. In
Fig. 4(b), the results at several fluences adjusted by the attenuators are summarized. In both cases shown in Figs. 4(a) and 4(b), the LIPSS period decreased with the number of macro-pulses. As shown in Figs. 4(a) and 4(b), $\Delta$ well obeyed the power law $\ln \Delta=a+b \ln N$. This power law was valid regardless of the fluence and excitation wavelength in this study. The values of the power-exponent $b$ were within a range of $-0.06<b<-0.1$. This almost constant exponent for the power law implies that a scaling law exists between $\Delta$ and $N$. Although a few other studies considering the pulse number dependence of the LIPSS period on Si are available, they did not consider the power law dependence. A similar power law was elucidated by redrawing the graphs in these studies [Figs. S2(a) ${ }^{17}$ and S2(b) ${ }^{7}$ in the supplementary material]. It should be noted that $b \approx-0.05$ was clearly observed for the power law in the case of LSFL on Si generated by an NIR fs-laser. ${ }^{16}$ The positive $b$ [Fig. S2(b) ${ }^{7}$ in the supplementary material] results from an unclassical ripple (U-ripple) caused by irradiation with a laser fluence above the meltingthreshold of Si. So far, all the LIPSS, including our findings, can be classified into several types, where the parameter for determining the LIPSS period was the wavelength, fluence, number of pulses, or some combination thereof. It is suggested that the parameter $b$ of the power law can also be classified into several types depending on the type of LIPSS.

The power law has also been observed for the accumulation effect beyond the damage-threshold of the fluence in the case of multiple-pulse irradiation by an Nd:YAG laser. ${ }^{18}$ In this case, the induced change in the damage threshold with the increase of irradiation pulse numbers can result in a
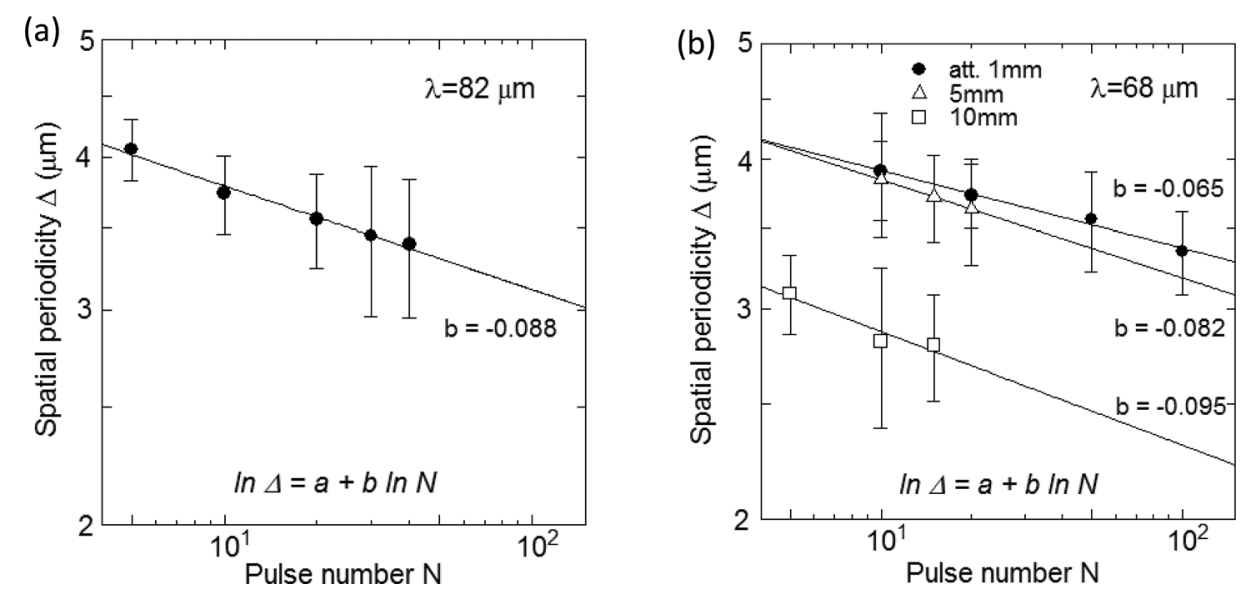

FIG. 4. Dependence of the LIPSS period on the number of macro-pulses at wavelengths of (a) 82 and (b) $68 \mu \mathrm{m}$. Solid circles in (a) show the data at a fluence of $32 \mathrm{~J} / \mathrm{cm}^{2}$. Open triangles, solid circles, and open squares in (b) show the data at fluences of 13 , 22 , and $32 \mathrm{~J} / \mathrm{cm}^{2}$, respectively. Values of $b$ in (a) and (b) are the results of power-law fits indicated by solid lines. 
change of the absorption energy by laser irradiation because the roughness of the crystal surface varies with repeated thermal stress-strain cycles as a function of the irradiation pulse number. The LIPSS formation mechanism should not be concluded to be a simple thermal effect demonstrated by the polarization dependence of irradiated lasers but should instead be considered the accumulation phenomena of both the laser damage threshold described above and the LIPSS period revealed in this study. From an energy transfer point of view, both phenomena indeed occur within the thermodynamic nonequilibrium open systems where various dissipative structures can emerge. ${ }^{19}$ So far, the term "self-organization" has been extensively used for the LIPSS formation phenomena, but this study demonstrates the scaling law governing the LIPSS formation mechanism and estimates the scaling exponents, i.e., the $b$ values in power-law fitting as shown in Figs. 4(a) and 4(b) [also Figs. S2(a)-S2(d) in the supplementary material, not only for Si but also for Ti plate and $\mathrm{Zr}$ alloy]. Imagining the self-organization as a physical mechanism of LIPSS generation, the possibilities of material functionalization can be pointed out from the viewpoint of fundamental material properties. The bottom-up type microfunctional material construction can be expected by forming a crystalline structure or a target compound structure on polycrystalline or amorphous or alloy materials. In fact, the LIPSS prepared in this study also gave results suggesting improvement of surface crystallinity of Si by Raman microscopy observation (unpublished). To elucidate the details of the power law and its physical origin, further investigations are needed for various materials over a wide wavelength range using IR and NIR lasers, mid-infrared (MIR)-FEL, and XFEL in addition to the THz-FEL.

Lastly, we would like to note the advantage of LIPSS studies that use THz-FEL. The period of the electric field oscillation of a THz-wave, i.e., far-infrared (FIR) light, is approximately 100 times longer than that of NIR. This means that the finer time-resolved changes in morphology and material properties, e.g., free carrier density, can be investigated by using THz-waves with pump-probe techniques. Such further studies using THz-waves are expected to provide advanced clues to resolve the universal mechanism of LIPSS formation.

In summary, we discovered that the LIPSSs on Si can be controllably generated by intense THz-FEL irradiation. The LIPSSs were almost parallel to the electric field of the linearly polarized THz-wave. The dependences of the LIPSS period on the fluence, wavelength, and macro-pulse number were investigated. Consequently, the minimum ratio of the LIPSS period to the excitation wavelength was $\sim 1 / 25$, which is four times smaller than that of typical HSFL generated by NIR fs-laser irradiation. These results are unable to be explained by the conventional models of LIPSS formation for the NIR fs-laser. On the other hand, the power law between the LIPSS period and the number of laser pulses was found not only in our experiment on Si using THz-FEL but also in other reports on various materials using an NIR fs-laser (see Refs. 7 and 17 and Refs. 20 and 21 in the supplementary material).

See supplementary material for additional data and discussion regarding the (1) outermost scratches with LIPSS observed for an irradiation with a small number of pulses; and (2) the scaling law on $\mathrm{Si}$ and other materials using an NIR fs-laser.

This work was supported by JSPS KAKENHI Grant No. JP17K18989, by the research program "Dynamic Alliance for Open Innovation Bridging Human, Environment and Materials," and by "Executive Program of Cooperation in The Field of Science and Technology between The Government of Italy and The Government of Japan (2017-2019).” Dr. K. Kawase, Dr. M. Fujimoto, Mr. K. Furukawa, Ms. K. Kubo, and Mr. Y. Okada supported us with the operation of THzFEL. Si wafers were kindly supplied by Professor K. Sakamoto. Professor S. Kimura helped us in the microscope observation. Professor A. Marcelli, Professor S. Lupi, and Professor S. Dabagov have encouraged us by useful discussions through the international collaboration.

${ }^{1}$ B. Wu, M. Zhou, J. Li, X. Ye, G. Li, and L. Cai, Appl. Surf. Sci. 256, 61 (2009).

${ }^{2}$ B. Dusser, Z. Sagan, H. Soder, N. Faure, J. Colombier, M. Jourlin, and E. Audouard, Opt. Express 18, 2913 (2010).

${ }^{3}$ M. Birnbaum, J. Appl. Phys. 36, 3688 (1965).

${ }^{4}$ F. Keilmann and Y. H. Bai, Appl. Phys. A 29, 9 (1982).

${ }^{5}$ T. Q. Jia, H. X. Chen, M. Huang, F. L. Zhao, J. R. Qiu, R. X. Li, Z. Z. Xu, X. K. He, J. Zhang, and H. Kuroda, Phys. Rev. B 72, 125429 (2005).

${ }^{6}$ A. Borowiec and H. K. Haugen, Appl. Phys. Lett. 82, 4462 (2003).

${ }^{7}$ W. Zhang, G. Cheng, and Q. Feng, Appl. Surf. Sci. 263, 436 (2012).

${ }^{8}$ J. Reif, O. Varlamova, S. Uhlig, S. Varlamov, and M. Bestehorn, Appl. Phys. A 117, 179 (2014).

${ }^{9}$ K. Kawase, R. Kato, A. Irizawa, M. Fujimoto, S. Kashiwagi, S. Yamamoto, F. Kamitsukasa, H. Osumi, M. Yaguchi, A. Tokuchi, S. Suemine, and G. Isoyama, Nucl. Instrum. Methods Phys. Res. Sect. A 726, 96 (2013).

${ }^{10}$ H. Hirori, K. Shinokita, M. Shirai, S. Tani, Y. Kadoya, and K. Tanaka, Nat. Commun. 2, 594 (2011).

${ }^{11}$ J. Bonse, S. Baudach, J. Krüger, W. Kautek, and M. Lenzner, Appl. Phys. A 74, 19 (2002).

${ }^{12}$ R. L. Harzic, F. Stracke, and H. Zimmermann, J. Appl. Phys. 113, 183503 (2013).

${ }^{13}$ T. Jia, F. Zhao, M. Huang, H. Chen, J. Qiu, R. Li, Z. Xu, and H. Kuroda, Appl. Phys. Lett. 88, 111117 (2006).

${ }^{14}$ Q. Z. Zhao, F. Ciobanu, S. Malzer, and L. J. Wang, Appl. Phys. Lett. 91, 121107 (2007).

${ }^{15}$ S. H. Kim, I. Sohn, and S. Jeong, Appl. Phys. A 102, 55 (2011).

${ }^{16}$ T. Iguchi, T. Sugaya, and Y. Kawano, Appl. Phys. Lett. 110, 151105 (2017).

${ }^{17}$ J. Bonse and J. Krüger, J. Appl. Phys. 108, 034903 (2010).

${ }^{18}$ Y. Jee, M. F. Becker, and R. M. Walser, J. Opt. Soc. Am. B 5, 648 (1988).

${ }^{19}$ N. Gregoire and I. Prigogine, Self-Organization in Nonequilibrium Systems (Wiley, New York, 1977), Vol. 191977. 